\title{
Arm Based Automated Wireless Greenhouse Climate Manage- ment System Using Zigbee Technology
}

\author{
Zoya Parvez $\mathrm{S}^{1}$, Fareduddin Ahmed J.S ${ }^{2}$, Shafreen Parvez $\mathrm{S}^{3}$, Gadilingappa $\mathrm{K}^{4}$ \\ ${ }^{I}$ (MTECH Student, Dept Of Ece, Bitm College, Bellary, (Vtu Belgaum), Karnataka, India) \\ ${ }_{2}^{2}$ (Assistant Professor, Dept Of Ece, Bitm College, Bellary, (Vtu Belgaum), Karnataka, India) \\ ${ }^{3}$ (Student, Dept Of Eee, Visvesvaraya Technological University, Belgaum, Karnataka, India) \\ ${ }^{4}$ (Student, Dept Of Eee, Visvesvaraya Technological University, Belgaum, Karnataka, India)
}

\begin{abstract}
Increases in greenhouse sizes have forced the growers to increase measurement points for tracking changes in the environment, thus enabling energy saving and more accurate adjustments. However, increases in measurement points mean increases in installation and maintenance cost. The purpose of this project "AUTOMATED WIRELESS GREENHOUSE CLIMATE MANAGEMENT SYSTEM" which is capable of intelligently monitoring and controlling the greenhouse climate conditions in a pre programmed manner.

The proposed system consists of three stations: Sensor Station, Coordinator Station, and Central Station. To allow for better monitoring of the climate condition in the greenhouse, the sensor station is equipped with several sensor elements such as CO2, Temperature, humidity, light, soil moisture and soil temperature. The communication between the sensor station and the coordinator station is achieved via ZigBee wireless modules and the communication between coordinator station and the central station is achieved via Zigbee wireless modules.

The overall system architecture shows advantages in cost, size, power, flexibility and distributed intelligent. It is believed that the outcomes of the project will provide the opportunity for further research and development of a low cost automated wireless greenhouse management system for commercial use.
\end{abstract}

Keywords: Green house, Zigbee, ARM7LPC 2148, Sensors

Green house history:

\section{Introduction}

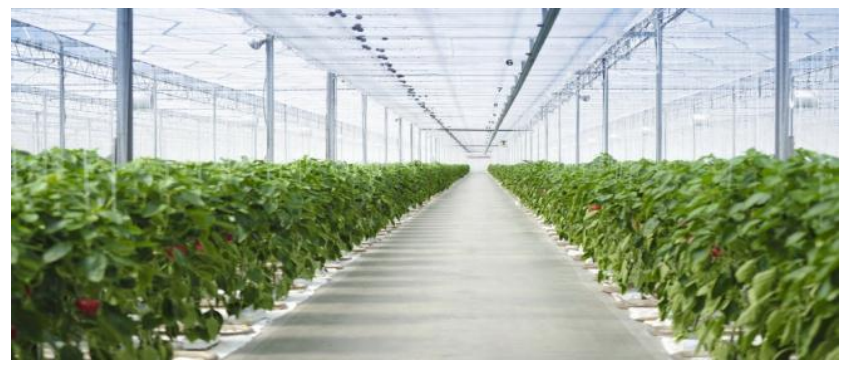

Crop cultivation has been around for a longtime. It plays a crucial role in the continuous development of human civilization. Tradition crop cultivation requires a tremendous amount of hard work and attention and there are several disadvantages in implementing traditional cultivation techniques:

- Weather dependent factors: plants" growth and development are primarily governed by the weather conditions.

- Pests and diseases: plants growing under traditional cultivation technique are significantly affected by pests and diseases.

It was discovered that there are indications that already many thousands years ago civilizations in countries such as China, Egypt and India employed means of protection against cold, wind and excessive solar radiation [10]. These methods of protection were employed only to provide a short-term protection for plants against harsh climate conditions. However, no further development occurred until the late 15th century and early 16th century, when European explorers brought back exotic plants acquired in the course of their travels. Many were tropical plants that could not endure the cold European climates. The result was the creation of greenhouses and these early greenhouses were originally referred to as "giardini botanic" also known as "botanical gardens" [11].

A Greenhouse represents a strange kind of agricultural environment characteristics by the presence of several input output devices and control devices. Requirement of human involvement is less compared to open air agriculture for providing water for irrigation and manures. Greenhouse represents a closed environment which can be strictly controlled by human in order to provide best conditions for the growth of plants. Wireless sensor 
networks (WSN) in agriculture have recently received great attention, and their use has been intensely investigated. Zigbee wireless technology is capable of providing large scale low power network and devices that could run for years on inexpensive batteries.

By the use of sensors, it is possible to monitor several environmental parameters such as

- TEMPERATURE

- HUMIDITY

- LIGHT

- CARBON-DI-OXIDE(Gas sensor)

- SOIL MOISTURE

Knowledge of these parameters helps the farmers perform the most suitable operations in order to improve the growth of plants and productivity and low cost, crops are grown within a short time duration in greenhouse.

\section{Objectives}

The aim of the Green Houses project was to achieve a 15\% reduction in overall energy consumption of participating households. The project objectives were to:

- Increase knowledge and awareness of energy issues and environmental impacts of energy use.

- Enable to identify energy wastage in their homes and adopt behaviors to reduce this wastage.

- Set goals to reduce their energy consumption and assist them in establishing strategies to make the lifestyle changes required to attain their goals.

- Identify successful communication techniques suitable for a large project that transfers knowledge and feedback to the participant.

\section{Literature Survey}

[1] Jia Song, He proposed a system on Greenhouse Monitoring and Control System using 8051 controller.[2] He,Guomi Wang, Xiaochan; Sun, Guoxiang .They had discussed about humidity and moisture monitoring in green house using Zigbee monitoring system.[3] In this project we have discussed about Greenhouse Monitoring and Control System Base on Zigbee Wireless Senor Network using ARM controller. The parameters in the green house are monitored on the PC. In this project we have proposed a low cost method using ARM microcontroller and Zigbee technology to accurately monitor and control the various parameters like humidity, soil moisture, light intensity carbon dioxide (gas sensor) and temperature. We are using Zigbee; transmit the message to the user's pc.

\section{Necessity}

It has been identified that the rate of photosynthesis depends on the amount of light energy falling on to the leaves as light intensity reduces the photosynthesis rate reduce and respiration rate increases therefore to get the maximum out of the plants food production the photosynthesis rate needs to be kept high in day time but it is impossible to control if the plant is grown outside a greenhouse. Temperature of the atmosphere can drop to values below the optimum temperature and reduce the rates of plants' respiration and photosynthesis rates and when the temperature keeps at a dropped value from the optimum value the yield would be less if the plants are subjected to natural environmental conditions. It is to be understood the terms photosynthesis and respiration. Photosynthesis is the process by which food is made by plants. They absorb $\mathrm{CO} 2$ and $\mathrm{H} 2 \mathrm{O}$ from the atmosphere and uses light energy to convert them to glucose molecules and store as glycogen.

Oxygen is formed as a by-product in this process. Respiration is the process by which food (organic molecules) is broken down to release $\mathrm{CO} 2, \mathrm{H} 2 \mathrm{O}$ and energy. these processes depends uses enzymes for the operations and the enzymes depend on temperature. At high temperature around $50^{\circ} \mathrm{C}$ the enzymes are denatured and at low temperatures they are inactive. Therefore controlling temperature is an essential need in a greenhouse. Due to the above mentioned processes in a plant a need for a controlled temperature, light intensity, humidity level, soil moisture, and carbon dioxide is required and it is has to done by an automated system

\section{COMPARISON WITH OTHER WIRELESS TECNOLOGY}

\begin{tabular}{|l|l|l|l|}
\hline Par ameters & ZigBee & Bhuetooth & Wi-Fi \\
\hline Siandard & 802.15 .4 & 802.15 .1 & $802.11 \mathrm{~b}$ \\
\hline $\begin{array}{l}\text { Memory } \\
\text { Requirement }\end{array}$ & $4-32 \mathrm{~KB}$ & $250 \mathrm{~KB}+$ & $1 \mathrm{MB}+$ \\
\hline Battery Life & Years & Days & Hours \\
\hline Data Rate & $250 \mathrm{Kbps}$ & $1-3 \mathrm{Mbps}$ & $11 \mathrm{Mbps}$ \\
\hline Range & $300 \mathrm{~m}$ & $10-100 \mathrm{~m}$ & $100 \mathrm{~m}$ \\
\hline
\end{tabular}




\section{Methodology}

The methodology used in this paper is that the system is an embedded system which will closely monitor and control the microclimatic parameters of a greenhouse on a regular basis round the clock for cultivation of crops or specific plant species which could maximize their production over the whole crop growth season and to eliminate the difficulties involved in the system by reducing human intervention to the best possible extent. This method ensures $100 \%$ yield with minimum use of chemical pesticides and disease free crops and plants

\section{Problem Definition}

- Complexity involved in monitoring climatic parameters like humidity, soil moisture, Illumination, soil pH, temperature, etc which directly or indirectly govern the plant growth.

- Investment in the automation process are high, as today's greenhouse control systems are designed for only one parameter monitoring (as per GKVK research centre); to control more than one parameter simultaneously there will be a need to buy more than one system.

- High maintenance and need for skilled technical labour. The modern proposed systems use the mobile technology as the communication schemes and wireless data acquisition systems, providing global access to the information about one's farms. But it suffers from various limitations like design complexity, inconvenient repairing and high price. Also the reliability of the system is relatively low, and when there are malfunctions in local devices, all local and tele data will be lost and hence the whole system collapses. More over farmers in India do not work under such sophisticated environment and find no necessity of such an advanced system, and cannot afford the same. Keeping these issues in view, a microcontroller based monitoring and control system is designed to find implementation in the near future that will help Indian farmers.

\section{Proposed System}

An attractive thing of our proposed system is that, it has extension in its concepts in an successful way. Here we have combined all available previous concepts for an effective and good productivity of plants. Both automatic controlling (Arm7 based) and manual controlling (zigbee wireless network based) takes place here. Automatic controlling process takes place continuously, when any of input module ie., sensors does not work properly required actions is not get performed. At that time we can use zigbee wireless sensor network based controlling process. The upper lines which I have denoted in bold is an great and successful changes made in this paper

\section{DESIGN SPECIFICATIONS:}

VII. System Overview:

Before the system can be developed and implemented, it is important to specify the design specifications and requirements necessary for the development of the system. This section explains these design specifications and requirements in detail.

\section{CONTROLLING STATION REQUIREMENTS:}

The central station needs to be able to

1. Send a data collection request to coordinator station

2. Send control instructions to coordinator station

3. Data processing

a) Data storage

b) Visually display real-time data

- CO-ORDINATOR STATION REQUIREMENTS:

The coordinator station needs to be able to

1. Receive requests and instructions from the controlling station

2. Collect a data packet from the sensor station and send it to the controlling station

3. Analyze the instruction packet

D If it's a data collection request, forward the instruction to sensor station

$>$ If it's a climate control request, send a signal to activate relay circuit

\section{SENSOR STATION REQUIREMENTS:}

The sensor station needs to be able to

1. Receive a data collection request from the coordinator station

2. Collect data from the environment

3. Perform analog to digital conversion on the collected data

4. Send data packets to the coordinator station 


\section{DESIGN CONSTRAINTS}

The following are the design constraints that needed to be considered:

1. The system must be compact and portable

2. The system must have low power consumption

3. The system must be reliable, robust and have a friendly user interface.

\section{Block Diagram}
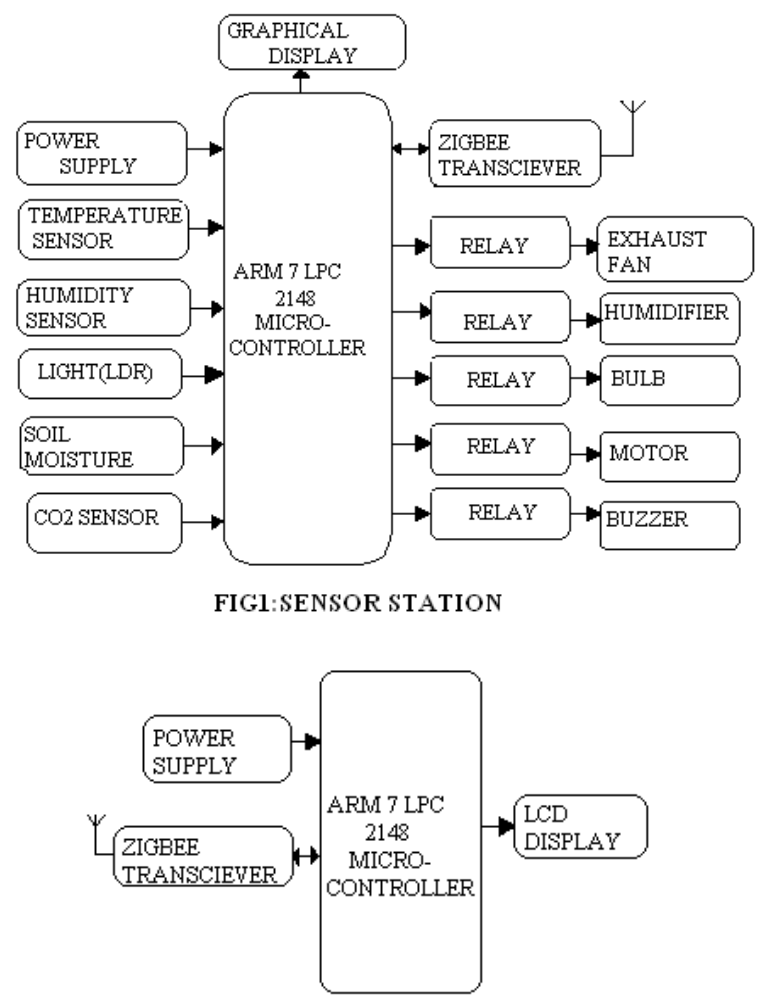

FIG2: CO-ORDINATOR STATION

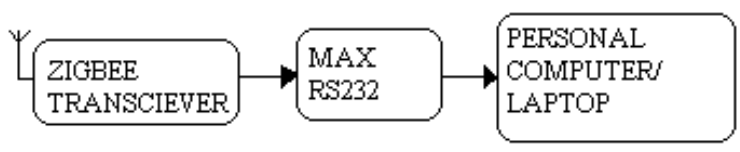

FIG3:CONTROLLING

STATION

\section{Block Diagram Description}

Here we are using an embedded systems approach to design an automated green house. Monitoring and Controlling of various environmental parameters artificially is of utmost importance to increase crop yield and productivity. The parameters are Light, Soil Moisture, Humidity, Temperature and carbon dioxide (smoke sensor) are monitored and controlled using ARM processor transmitted through the Zigbee and display the presence of parameters on LCD display. In this project there are three stations:

- SENSOR STATION

- COORDINATOR STATION

- CONTROLLING STATION

Here we are discussing about particular components that are used in this project. The details are as follows:

\section{A.ARM MICROCONTROLLER:}

The ARM7TDMI-S is a general purpose 32-bit microprocessor, which offers high performance and very low power consumption. The ARM architecture is based on Reduced Instruction Set Computer (RISC) principles, and the instruction set and related decode mechanism are much simpler than those of micro programmed Complex Instruction Set Computers (CISC). This simplicity results in a high instruction throughput and impressive real-time interrupt response from a small and cost-effective processor core. Pipeline techniques are employed so that all parts of the processing and memory systems can operate continuously. Typically, while one instruction 
is being executed, its successor is being decoded, and a third instruction is being fetched from memory. The LPC21xx microcontrollers are based on a 16-bit/32-bit ARM7 CPU with real-time emulation and embedded trace support, that combine microcontroller with embedded high speed flash memory. Advantages of using RISC machine

- Smaller die sizes

- Shorter time to develop

- Possible higher performance than CISC

\section{B. ZIGBEE:}

Zigbee is new wireless technology guided by IEEE 802.15.4 Personal Area Network standard. It is primarily designed for the wide range controlling applications and to replace the existing non-standard technologies. It currently operates in $868 \mathrm{MHz}$ band at a data rate of $20 \mathrm{Kbps}$ in Europe, $914 \mathrm{MHz}$ band at $40 \mathrm{kbps}$ in USA, and the $2.4 \mathrm{GHz}$ ISM bands Worldwide at a maximum data-rate of $250 \mathrm{kbps}$. It is used to verify whether user's truncation is possible or not. One of the main advantages of this ZIGBEE communication is that it provides a noise free communication, the amount of noise added in this type of communication is very less compared to the other wireless communications.

\section{SENSORS:}

There are four sensors namely temperature ,humidity, light and soil moisture interfaced to the ARM microcontroller, used to sense the environmental parameters. If the set value is altered it is controlled automatically and the information is sent to the user through Zigbee.

1. TEMPERATURE SENSOR: LM35 is used to sense different temperature values. This has a linear characteristic. This sensor gives a variable output voltage with respect to the temperature variations in a greenhouse. The LM-35 is used as temperature sensor which is a precision integrated circuit temperature sensor, calibrated directly in ${ }^{\circ}$ Celsius (Centigrade), Linear $+10.0 \mathrm{mV} /{ }^{\circ} \mathrm{C}$ scale factor with accuracy $0.5^{\circ} \mathrm{C}$ (at $+25^{\circ} \mathrm{C}$ ) with rating ranging from $-55^{\circ}$ to $+150^{\circ} \mathrm{C}$ range. Here we have to set the minimum temperature and maximum temperature values.

2. LDR (LIGHT DEPENDENT RESISTOR): It is very useful especially in light/dark sensor circuits. Normally the resistance of an LDR is very high, sometimes as high as $1000000 \mathrm{ohms}$, but when they are illuminated with light resistance drops dramatically, allowing current to pass through it.

3. HUMIDITY SENSOR: HYSH 220 humidity sensor is used to sense the humidity values, it coverts relative humidity to voltage. The sensor output is a variable voltage with respect to humidity level and expressed in terms of $\%$. The SY-HS-220 humidity sensor is used which converts relative humidity to the output voltage with operating humidity range $30 \%-90 \% \mathrm{RH}$ and accuracy is $\pm 5 \% \mathrm{RH}\left(\right.$ at $\left.+25^{\circ} \mathrm{C}\right)$. In normal condition the humidity will be around $50 \%$ to $70 \%$. Here, we have to set the minimum humidity and maximum humidity values.

4. SOIL MOISTURE SENSOR: Here we are connecting a moisture based Electrode sensor. As soon as the water dries up then the electrode voltage rises to $5 \mathrm{v}$ which is applied to the non inverting terminal and the output of the amplifier is $0 \mathrm{v}$ which will in turn, turn on the AC motor. When the water level is up then the electrode voltage drops to $0 \mathrm{v}$ which is applied to the non inverting terminal and the output of the amplifier is $5 \mathrm{v}$ which will in turn, turn off the AC motor.

5. CARBONDIOXIDE SENSOR(CO2 SENSOR)

This sensor is used to sense the concentration of $\mathrm{CO} 2$ in a greenhouse. This sensor is also used as gas sensor to detect the smoke if occurs in greenhouse then the buzzer is activated. Sensitive material of MQ-3 gas sensor is $\mathrm{SnO} 2$, which with lower conductivity in clean air. MQ-3 gas sensor has high sensitivity to Alcohol, and has good resistance to disturb of gasoline, smoke and vapor. The sensor could be used to detect alcohol with different concentration; it is with low cost and suitable for different application.

\section{D.ACTUATORS:}

It is a device which is used to control the parameter to the desired level. The actuators used in the system are:

- Fan

- Heater

- De-humidifier

- Humidifier

- Bulb

- Motor

- Buzzer 


\section{E.POWER SUPPLY UNIT:}

The system needs regulated power supply which provides necessary voltages for proper working of its blocks. This unit is specially designed to gives out all necessary voltage levels required for working of all blocks

F.RELAYS: A relay is a simple electromechanical switch made up of an electromagnet and a set of contacts.

There are five relays connected. It operates in two modes:

Normally Open

Normally Closed

Different devices can be controlled i.e. they can be turned On/Off whenever required.

G.LCD (LIQUID CRYSTAL DISPLAY): LCD is used to display the prescription provided by the ARM microcontroller and is interfaced to the microcontroller.

H.RS232:

To allow compatibility among data communication equipment made by various manufactures, an interfacing standard called RS232 was set by the Electronic Industries Association (EIA). This RS-232 standard is used in PCs and numerous types of equipment.However, since the standard was set long before the advent of the TTL logic family, its input and output voltage levels are not TTL compatible. In RS-232, a 1 is represented by -3 to $25 \mathrm{~V}$, while a 0 bit is +3 to $+25 \mathrm{~V}$, making -3 to +3 undefined. For this reason, to connect any RS -232 to a microcontroller system we must use voltage converters such as MAX232 to convert the TTL logic levels to the RS232 voltage levels and vice versa.

So here we are using this MAX-232 to have compatibility between the PC and microcontroller.

\section{Results:}

After the feasible results obtained for green house monitoring and controlling which is displayed in LCD display and in PC/Laptop. A hardware kit has been developed and put it on the green house environment. The kit consists of a power supply unit, various sensors, Arm the output from the sensors is continuously given to the Arm. Simultaneously the display is reading in LCD module. In the real application the entire kit it is fixed on the green house system. When the parameters like temperature, humidity, soil wetness and light intensity are varied from set parameters, which is continuously monitored and controlled with the help of personal computer or laptop.

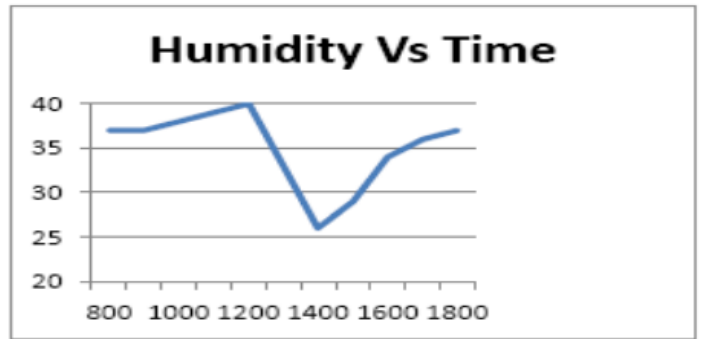

Fig4: Humidity vs Time

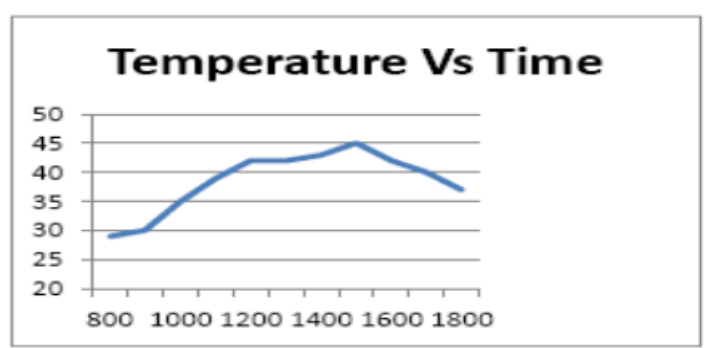

Fig5: Temperature vs time

\section{Software Results ALGORITHM}

Algorithm for Greenhouse automation is as follows.
1. Start
2. Initialize ADC.
3. Initialize LCD.
4. Initialize UART
5. Get all set points from EEPROM
6. Get feedback from Temperature sensor 
7. If temperature $>$ Set temperature then turn ON the Cooler (FAN)

8. If temperature < Set temperature then turn ON the Heater

9. Get feedback from Humidity sensor

10. If humidity > Set humidity then turn ON the Humidifier

11. If humidity < Set humidity then turn ON the Dehumidifier

12. Get feedback from LDR

13. If light intensity < Set light intensity then turn ON the Bulb

14. If light intensity > Set light intensity then turn OFF the Bulb

15. Get feedback from Soil moisture sensor

16. If soil moisture < Set soil moisture then turn ON the Motor

17. If soil moisture > Set soil moisture then turn OFF the Motor.

18. Get feedback from carbon dioxide $\left(\mathrm{CO}_{2}\right)$ sensor.

19. If $\mathrm{CO}_{2}>$ set $\mathrm{CO}_{2}$ then buzzer turn $\mathrm{ON}$ alert alarm

20. If $\mathrm{CO}_{2}<$ set $\mathrm{CO}_{2}$ then buzzer turn OFF alert alarm.

21. If data received from $\mathrm{PC}$, if $\mathrm{NO}$ go to , $6^{\text {ec }}$

22. If data received $=, I^{e}$ then send all info to $\mathrm{PC}$ and go to , $6^{\text {"e }}$

23. If data received $=, T^{\prime \prime}$ then update set temperature and go to , $6^{\text {ce }}$

24. If data received $=, \mathrm{H}^{\text {e }}$ then update set humidity and go to , $6^{\text {ee }}$

25. If data received $=, \mathrm{L}^{\mathrm{Ce}}$ then update set light intensity and go to, $6^{\mathrm{ce}}$

26. Go to , $6^{\text {ce }}$

27. Stop.

\section{Advantages and Disadvantages}

\section{ADVANTAGES}

1. It has the ability to monitor five different greenhouse climate parameters (temperature, relative humidity, light intensity, carbon dioxide, and soil moisture).

2. The system does not need cables to run and has low power consumption.

3. It comprises embedded wireless sensor nodes that can be used to collect real time environmental data.

4. It allows communication between the controlling station and actuators that are located in different part of green house.

5. Moreover, the system is easy to relocate once installed and maintenance is relatively cheap and easy. The only additional cost occurs when the batteries run out.

6. The system is scalable - more measurement points can be added to the system at only a fraction of the cost

7. The system is flexible and reliable

\section{DISADVANTAGES}

1. Complete automation in terms of pest and insect detection and eradication cannot be achieved.

2. No self-test system to detect malfunction of sensors.

\section{Applications}

1. Greenhouse can be implemented for irrigation purpose and agricultural field for the betterment of crops growth

2. For commercial market that is to obtain wide variety of crops in large amounts in all seasons.

3. Plants of medicinal values are grown under controlled greenhouse environment.

\section{Conclusion}

In this paper automated greenhouse control is discussed with advantages of low cost and accuracy. The benefits that a fully automated greenhouse control systems bring to the grower are many. Obviously, there will be the labour saving aspect but far more importantly, factors such as improved quality of product and information gathering can mean the difference between earning a profit or suffering substantial losses. Greenhouse prevents the plant from the effects of climate; insects and so on, which makes great sense for agricultural production. The automation and high efficiency on greenhouse environment monitoring and control are crucial. If the parameters are within the range then the value is displayed in the LCD. If it is not within the range the controller will turn on/off the actuators as per the requirements. Thus controlling the Greenhouse automatically without human intervention. The system not only saves the energy consumption significantly, but also reduces a large number of inputting on the human and material resources in the management. Applying embedded technology and ZigBee wireless transceiver technology to the rapid deployment system of the incident detection of 
emergency food storage environment without complicated connections, it enhances the system's flexibility, small size, low cost and good effective, so it is easy to install and migrate.

\section{Future Scope}

- This system can be connected to communication devices such as modems, cellular phones or satellite terminal to enable the remote collection of recorded data or alarming of certain parameters.

Time bound administration of fertilizers, insecticides and pesticides can be introduced.

- A speaking voice alarm could be used instead of the normal buzzer

- More sensors can be added to the sensing unit to monitor others environmental parameters such as soil pH level, carbon monoxide $(\mathrm{CO})$ and oxygen $(\mathrm{O})$ level.

- Global System for Mobile Communication (GSM) and Short Message Service (SMS) can also be integrated into the system. These extra features will allow the system to directly alert the user of any abnormal changes in the greenhouse environment through the transmission of a simple short text message.

\section{REFERENCES}

[1] Jian Song, "Greenhouse Monitoring and Control System Based on Zigbee Wireless Senor Network" ICECE '10 Proceedings of the 2010 International Conference on Electrical and Control Engineering IEEE Computer Society Washington, DC, pp.2785-2788 USA (C) 2010 .

[2] He,Guomi Wang, Xiaochan; Sun, Guoxiang, " Design of a Greenhouse Humiture Monitoring System Based on ZigBee Wireless Sensor Networks"Fifth International Conference on Frontier of ComputerScience and Technology (FCST), 2010 . [3]Anuj Kumar, Abhishek Singh, I.P.Singh,S.K.Sud, "Prototype Greenhouse Environment Monitoring System" vol .II Mar 17-19 IMECS 2010.

[4] BeomJin Kang Dae, Heon Park, KyungRyung Cho, ChangSun Shin, SungEonCho, JangWoo Park "A Study on the Greenhouse Auto Control System Based on Wireless Sensor Network", IEEE, 22 December 2008.

[5] Huichun Xing, Jing Li, Richard Liu, Ed Oshinski, Richaerd Rogers, "2.4 GHZ On-Board Parallel Plate Soil Moisture Sensor system", Sensors for Industry Conference Houston, Texas, USA, 8-10 February 2005.

[6] CC2420 $24 \mathrm{GHz}$ IEEE 802.15.4/Zigbee RF Transceiver. Available online: http://www.alldatasheet.com

[7] S.R. Bhagya Shree, ManojKollam"ZigbeeWirelessSensor Network for better interactive industrial automation", IEEE ICoAC 2011.

[8]" Digital Communication fundamental and application "By Bernard Sklar, second edition,Pearson Education (page no: 256-259).

[9] http://documents.rscomponents.com/EITC/WW/TandM/Tekronix\%20 $\% 20$ Power\%20Supply\%20Measurement $\% 20 \& \% 20$ Analysis.pdf.

[10] Bakker, J.C., Greenhouse climate control: an integrated approach, ed. J.C. Bakker.1995, Wageningen Wageningen Pers.

[11]. Katemopoulos, M. The History of the Greenhouse. 2009 [cited 2010 8/9]; Available from: http://sharonfalsetto.suite101.com/history-of-the-greenhouse-a81808

\section{AUTHOR 1: ZOYA PARVEZ S}

\section{BIBLOGRAPHY}

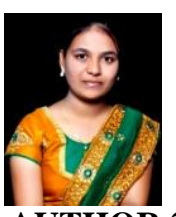

Pursuing MTECH (ECE) student of BITM College,BELLARY,Karnataka and completed BE(EEE) in RYMEC College ,BELLARY, Karnataka of Visvesvaraya Technological University,Belgaum,Karnataka,INDIA.area of interest in embedded systems, vlsi design, electrical power generation,DC machines.

\section{AUTHOR 2: FAREDUDDIN AHMED.J.S}

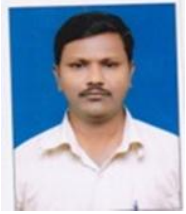

Obtained MTECH (CNE) in BITM COLEGE, BELLARY, Karnataka, INDIA and obtained BE (ECE) in RYMEC, BELLARY, Karnataka of Visvesvaraya Technological University, Belgaum, Karnataka, INDIA working as Assistant Professor in BITM COLLEGE, BELLARY. With working experience of 14 years in teaching field worked as Associate Professor \& HOD of CSE dept with area of interest in embedded systems, operating systems, optical fibre.

\section{AUTHOR 3: SHAFREEN PARVEZ S}

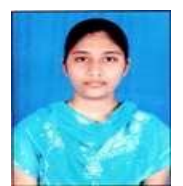

Completed BE (EEE) in RYMEC College, BELLARY, Karnataka of Visvesvaraya Technological University, Belgaum, Karnataka, INDIA.with area of interest in electrical power generation, digital signal processing, and transformer induction machines.

\section{AUTHOR 4: GADILINGAPPA K}

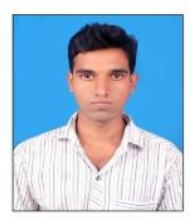

Completed BE(EEE) in RYMEC College,BELLARY,Karnataka of Visvesvaraya Technological University,Belgaum,Karnataka,INDIA.with area of digital electronics, power electronics, power systems,drives.working as junior engineer in KPTCL,Karnataka,INDIA. 